\title{
Hellenic Woodland Database
}

\author{
Georgios Fotiadis, loannis Tsiripidis, Erwin Bergmeier \& Panayotis Dimopolous
}

\begin{abstract}
The Hellenic Woodland Database (GIVD ID EU-GR-006) includes relevés from 59 sources, approximately, as well as unpublished relevés. In total 4,571 relevés have already been entered in the database, but the database is going to continue growing in the near future. Species abundances are recorded according the 7-grade Braun-Blanquet scale. The oldest relevés date back to 1963. For the majority of relevés (more than 90\%) environmental data (e.g. altitude, slope aspect, inclination) exist. Relevés entered in the database represent both evergreen and deciduous forests, as well as scrubs and they belong (according to the original publications) to the classes Quercetea ilicis, Rhamno-Prunetea spinosae, Erico-Pinetea, Quercetea pubescentis, Querco-Fagetea, Vaccinio-Piceetea and Populetea albae. Most relevés (approximately 69\%) concern northern Greece (floristic regions of northeastern, north-central Greece and northern Pindos), while the remainder concern the rest of Greek mainland as well as the islands.
\end{abstract}

Keywords: beech; conifer; macchia; oak; riparian vegetation; silbjak.

\section{Hellenic Woodland Database}

Scope: Classification of forest communities and study of forest ecology

Status: [NA]

Period: $1963-2006$

Database manager(s): Georgios Fotiadis (gfotiad95@gmail.com); loannis Tsiripidis (tsiripid@bio.auth.gr)

Owner: Georgios Fotiadis, loannis Tsiripidis, Erwin Bergmeier, Panayotis Dimopoulos

Web address: [NA]

Availability: according to a specific agreement

Online upload: no

Online search: no

Database format(s): SORT $4 \quad$ Export format(s): Excel

Publication: Tsiripidis I. Fotiadis G. Karagiannakidou V. \& Babalonas D. 2005. Classification problems of forest vegetation in Greece: Transition from beech to deciduous oak zone. Botanica Chronika 18 (1): 253-268.

Plot type(s): normal plots

Non-overlapping plots: 4,571

Total plot observations: 4,571

Countries: GR: $100.0 \%$

Forest: $100 \%$ - Non-forest: aquatic: $0 \%$; semi-aquatic: $0 \%$; arctic-alpine: $0 \%$; natural: $0 \%$; semi-natural: $0 \%$; anthropogenic: $0 \%$

Guilds: all vascular plants: $100 \%$

Environmental data: altitude: $94 \%$; slope aspect: $91 \%$; slope inclination: $92 \%$; microrelief: $31 \%$; other soil attributes: $12 \%$

Performance measure(s): cover: $100 \%$

Geographic localisation: GPS coordinates (precision $25 \mathrm{~m}$ or less): $12 \%$; small grid (not coarser than $10 \mathrm{~km}$ ): $63 \%$; political units or only on a coarser scale (>10 km): $25 \%$

Sampling periods: 1960-1969: 6.1\%; 1970-1979: 12.9\%; 1980-1989: 18.3\%; 1990-1999: 35.4\%; 2000-2009: 27.4\% Information as of 2012-07-12; further details and future updates available from http://www.givd.info/ID/EU-GR-006

Georgios Fotiadis* (gfotiad95@gmail.com)

Department of Forestry and Management of Natural Environment, Technological Education Institute of Lamia, GR-36100 Karpenisi, GREECE

Ioannis Tsiripidis (tsiripid@bio.auth.gr)

School of Biology, Aristotle University of Thessaloniki, GR-54124 Thessaloniki, GREECE

Erwin Bergmeier (erwin.bergmeier@bio.uni-goettingen.de)

Department of Vegetation and Phytodiversity Analysis, Albrecht von Haller Institute of Plant Sciences, University of Göttingen, Untere Karspüle 2, DE-37073 Göttingen, GERMANY

Panayotis Dimopoulos (pdimopul@uwg.gr)

Department of Environmental and Natural Resources Management, University of Western Greece, Seferi 2, GR-30100 Agrinio, GREECE

*Corresponding author 